\title{
Near-field optical imaging of noble metal nanoparticles
}

\author{
G.P. Wiederrecht ${ }^{\mathrm{a}}$ \\ Chemistry Division and Center for Nanoscale Materials, Argonne National Laboratory, Argonne, IL 60439, USA
}

Received: 15 June 2004 / Accepted: 1st July 2004

Published online: 13 August 2004 - (C) EDP Sciences

\begin{abstract}
A review of the subject of near-field optical imaging of metal nanoparticles is presented. The emphasis of the review is on experimental achievements in optical imaging of single metal nanoparticles and nanostructured arrays with metal nanoparticle building blocks. The importance of the measurements as they relate to nano-optical applications and scientific understanding of optical near-fields is highlighted. Advancements in scanning near-field optical microscopy (SNOM) that have enabled near-field images of these structures are also featured, as are theoretical contributions for interpreting SNOM images.
\end{abstract}

PACS. 07.79.Fc Near-field scanning optical microscopes - 78.67.Bf Nanocrystals and nanoparticles 71.45.Gm Exchange, correlation, dielectric and magnetic response functions, plasmons

\section{Introduction}

The "far-field" optical properties of noble metal nanoparticles have fascinated artisans and scientists throughout modern history. The Romans, though likely without realizing it, used metal nanoparticles to create rich colors in stained glass. A famous example is the Lycurgus Cup of the 4th Century, which is green when viewed in reflected light but red when light is transmitted through the glass [1]. Analysis of the cup revealed the presence of $\mathrm{Ag}$ and $\mathrm{Au}$ nanoparticles to produce the unusual optical properties $[2,3]$. Noble metal nanoparticles also played a central role in luster pottery, beginning in the 9th century and reaching a pinnacle in Italian majolicas of the 15 th and 16 th centuries $[2,3]$. These materials inspired the great 19th century British scientist Michael Faraday, who realized the true state of the metals in these artworks by recognizing that the metal salts used in gold sols actually were "reduced in exceedingly fine particles, which becoming diffused produce a beautiful ruby red fluid. . . containing in fact no dissolved gold but only diffused gold" $[4,5]$. He went on to report the size dependence of nanoparticle optical properties, explaining that "a mere variation in the size of its particles gave rise to a variety of colors".

While these far-field visual attributes have been explained and quantified to a large extent, we are only in the initial stages of appreciating the similarly rich properties of the optical near-fields of noble metal nanoparticles, i.e. those electromagnetic fields that extend only a few nanometers from the metal surface. Much of the impetus for excitement related to these evanescent optical fields of metal nanoparticles is driven by the discovery and development of surface enhanced Raman scattering (SERS) that can now produce single molecule

\footnotetext{
a e-mail: wiederrecht@anl.gov
}

spectroscopies [6-12]. Evanescent fields of metal nanoparticles are primarily the result of the large scattering and absorption cross sections that result from the surface plasmon resonance. This is the response of free (conduction band) electrons that oscillate in concert with an incident electromagnetic wave [13]. The localized optical field that results on the surface of the nanoparticle can be enhanced by several orders of magnitude over the incident field, thus the SERS response. Quantitatively, the extinction cross section $\left(C_{\text {ext }}\right)$ of metal nanoparticles relates to the complex dielectric function behavior of metals [14] and is predicted by Mie theory [15] to be [1]:

$$
C_{e x t}=\frac{24 \pi^{2} R^{3} \varepsilon_{m}^{3 / 2}}{\lambda} \frac{\varepsilon^{\prime \prime}}{\left(\varepsilon^{\prime}+2 \varepsilon_{m}\right)^{2}+\varepsilon^{\prime \prime 2}}
$$

where $\varepsilon^{\prime}$ is the real dielectric constant, $\varepsilon^{\prime \prime}$ is the imaginary dielectric constant, $\varepsilon_{m}$ is the surrounding medium's dielectric constant, $R$ is the nanoparticle radius and $\lambda$ is the illumination wavelength. For the noble metals, $\varepsilon^{\prime}$ is negative throughout the visible spectral region and $\varepsilon^{\prime \prime}$ is weakly positive. As can be seen from this relation, when $\varepsilon^{\prime}=-2 \varepsilon_{m}$, a plasmon peak in the extinction cross section occurs. The enormous extinction coefficients of $10 \mathrm{~nm}$ diameter noble metal nanoparticles are in the range of $10^{7} \mathrm{M}^{-1} \mathrm{~cm}^{-1}$, or about two orders of magnitude stronger than is typical for organic laser dye molecules. For silver, the plasmon peak in air occurs in the near UV at approximately $360 \mathrm{~nm}$, while for Au the peak occurs at approximately $425 \mathrm{~nm}$ [13]. However, as predicted by equation (1), the higher dielectric constants of solvents or substrates produces a significant red shift of these plasmon resonances by tens or even hundreds of nanometers.

In addition to SERS, the evanescent fields of metal nanoparticle structures produce a range of physical 
and chemical phenomena that impact an extraordinary breadth of basic science research and technological applications. Potential applications include optical computing, high-bandwidth all optical telecommunications routing, high-density optical data storage, and nanoscale sensors-on-a-chip to name a few. In order to enable further nanophotonic and nanoelectronic applications, precise characterization of the spatial profile of these fields is necessary and this research area is the subject of the current review. As an example, the spatial profile of the near-field of metal nanoparticles impacts the efficiency of near-field energy exchange in arrays of closely spaced metal nanoparticles that are of interest for sub-diffraction limited waveguides. In these one dimensional arrays, electromagnetic energy propagation occurs through the propagation of surface plasmon polaritons, which are photon coupled free electron oscillations in metals [16-20]. Interest in spatially mapping nanoparticle-light interactions is further driven by advances in chemical synthesis and lithographic techniques that reliably produce complex shapes, narrow size distributions, and hybrid core-shell materials. These efforts make clear that the optical properties of metal nanoparticles can be tailored to facilitate a desired photochemical or photophysical response. Sub-wavelength near-field spatial resolution is critical for understanding, optimizing, and developing these unique responses at the nanoscale.

To gain improved understanding of these interactions, a variety of optical microscopies have been undertaken [21-26]. For this review, we will focus on an increasingly widely used and versatile field of scanning near-field optical microscopy (SNOM). This technique has been widely developed in the past twenty years to overcome the spatial resolution limits of conventional optical microscopes. These microscopes, classified as scanning probe microscopes, have since proven their utility in various nanoscopic applications. Their purpose is to characterize electromagnetic fields or modify matter at the sub-wavelength scale, as well as provide a unique way to understand the interactions between objects and fields at the nanometric scale. In this review, we begin by briefly introducing the basic principles behind SNOM, as well as some of the basic configurations for SNOM studies. This is followed by a review of near-field optical studies of single metal nanoparticles and advanced techniques that have added significant scientific utility in the past few years to the SNOM method. The collective interactions and imaging of metal nanoparticle arrays are then reviewed. The use of metal nanoprobes that function as illumination sources through field enhancement, as well as theoretical considerations for modeling near-field images of metal nanoparticles are also reviewed.

\section{Experimental methods}

\subsection{SNOM techniques}

A variety of scanning probe techniques have been utilized to image metal nanoparticles with sub-wavelength
General principle of the SNOM : breaking the diffraction limit by putting a scanned nanoprobe in the near-field of the sample

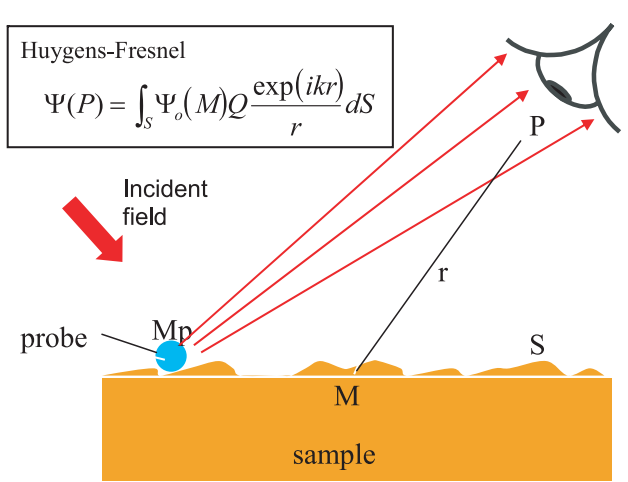

$>\lambda$ far-field (only propagating waves)

$<\lambda$ near-field (evanescent waves + propagating waves)

Fig. 1. The general principle of SNOM is shown. Far-field detection is always utilized to image the probe sample interaction. $\Psi(P)$ is sensitive to a variation of $\Psi_{o}\left(M_{p}\right)$, the optical field produced through dipole-dipole interactions between the sample and scanning probe.

resolution. These include aperture SNOM, apertureless (scattering) SNOM, and variations such as photon scanning tunneling microscopy (PSTM). All of these methods rely on a scanning probe methodology, in which a sub-wavelength object illuminates and/or scatters photons with sub-wavelength resolution, while the sample or probe is rastered to perform microscopy. Since a complete review of near-field imaging is beyond the scope of this paper, the reader is referred to excellent reviews by Dunn, or more recently, by Pohl $[27,28]$. There are also very comprehensive books on SNOM [29,30].

The general principal of SNOM relies on the introduction of a nanoparticle (namely a probe extremity) in the near-field of the sample to be studied (see Fig. 1 as an illustration). For a given position $M_{p}$ of the particle on the sample surface, the field $\Psi_{o}\left(M_{p}\right)$ resulting from the dipoledipole type interaction between $M_{p}$ and the probe strongly depends on the local optical properties of $M_{p}$ viewed by the probe. The total far-field collected at $P, \Psi(P)$, can be described by the Huygens-Fresnel principle (see inset, where $S$ is the surface over which the Huygens wavelets interact with each other). $\Psi(P)$ depends (slightly but certainly) on $\Psi_{o}\left(M_{p}\right)$ which represents a differential part of the Huygens-Fresnel integration.

In the case of far-field optical microscopy, all of the sample zones within the diffraction limited zone $S$ are fixed and $\Psi(P)$ is fixed as well. As a consequence, $\Psi(P)$ describes only the sample optical properties averaged over $S$ which can not be smaller than $\lambda / 2$. In the case of SNOM, the principle of Huygens-Fresnel is of course still valid, but in this case one gets the unique possibility to move the probe in a controlled way within the sample near-field. This allows $\Psi(P)$ to be sensitive to a variation of $\Psi_{o}\left(M_{p}\right)$ (where $M_{p}$ can be varied by probe scanning), that is to say the local properties of the sample viewed by the probe. In this way, scanning the probe onto the sample surface allows for the description, pixel by pixel, of the optical 


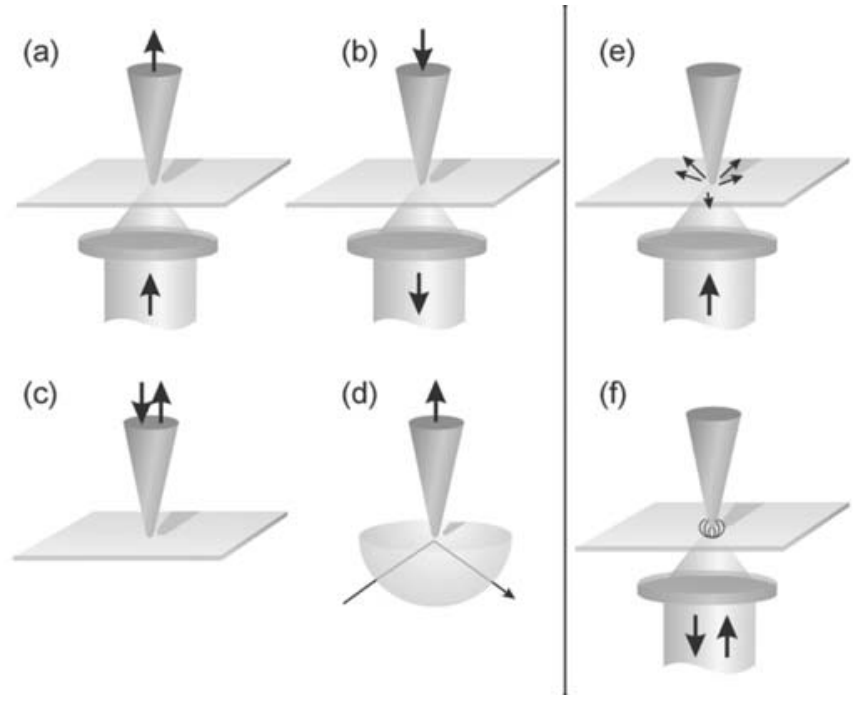

Fig. 2. The typical SNOM configurations available are shown. Configurations a-d are aperture based SNOM configurations, providing (a) collection mode, (b) illumination mode, (c) illumination/collection mode, and (d) collection mode using total internal reflection. Configuration (e) is for scattering SNOM using a dielectric tip, where illumination can be below or above the sample depending upon sample thickness or transparency. Configuration (f) is for metal probes, where field enhancement can occur at the apex of the probe. In this way, the metal probe can function as a confined light source, and even provide spatially confined SHG and white light generation.

properties of the surface and leads to a near-field optical image $I\left(M_{p}\right)$.

The nature of the SNOM probe configuration can vary widely as illustrated in Figure 2. The motivation for having many configurations is due to differences in samples (such as transparency and size) and the desired contrast mechanism (such as fluorescence, Raman, or evanescent field enhancement). Thus, the probe can be used either as a photon collector or as a local optical source. Also the probe can be a nanoaperture used to squeeze the electromagnetic field, leading to a decrease of $S$ and an increase of the contribution of $\Psi_{o}\left(M_{p}\right)$ in $\Psi(P)$. Alternatively, this can be accomplished with the extremity of an apertureless metal tip that acts as a nanoantenna that is locally sensitive to $M_{p}$. In this case, only sufficiently strong near-field effects allow $\Psi_{o}\left(M_{p}\right)$ to be extracted from the total $\Psi(P)$.

It should be stressed that, whatever the nature of the probe, the general principle presented above remains valid. With this in mind, we review below the basic SNOM configurations that are currently used.

\subsubsection{Aperture SNOM}

The basis of modern aperture SNOM was proposed in a series of papers by E.H. Synge in the 1920s and 1930s [31-33]. In these seminal papers, Synge proposed that light be incident on a sub-wavelength hole in an opaque screen and that the screen be placed close enough to the sample to avoid diffraction effects of the aperture. Thus, Synge predicted the SNOM microscope decades before it became technically feasible. The modern SNOM was first demonstrated by Pohl and colleagues in 1984 [34]. Here, the advancement came from the method for making a small aperture in a metal coated quartz probe and the ability to spatially resolve optical fields of a $400 \mathrm{~nm}$ chromium grating structure.

Today, the aperture technique is most commonly achieved through the creation of a small aperture in a tapered fiber, generally created with a fiber puller [27]. Usually, gold or aluminum is coated onto the fiber to increase optical throughput. Focused ion beam (FIB) milling can be used to create higher quality and more reproducible apertures. Figures $2 \mathrm{a}-\mathrm{d}$ illustrate the most generally applied configurations for aperture SNOM today. Configuration (a) is for "collection mode" SNOM, whereby the sample is illuminated with diffraction limited optics and near-field optical information is collected by the fiber probe. Configuration (b) illustrates "illumination mode" SNOM. Here, opposite to that of (a), a sub-diffraction limited area is illuminated and collection occurs with diffraction limited optics. Configuration (c) shows illumination/collection SNOM and is useful for highly scattering or thick samples. Finally, (d) illustrates collection mode SNOM using total internal reflection. This configuration is useful for removing a large fraction of the illumination light from the background, and is also used for plasmonic studies of metal films in order to match the wavevector requirements of the surface plasmon polariton dispersion relation [35].

\subsubsection{Apertureless SNOM}

This technique originated with the proposal of Wessel, in which he envisioned that the confined optical fields of a nanometric metallic particle could be used as a near-field probe [36]. In the current state of apertureless SNOM, a dielectric, metallic, or semiconductor probe can be used to scatter near-field photons to a far-field detector. Experimental demonstration of apertureless SNOM was initiated nearly simultaneously by three different research groups [37-39]. An example of the apertureless configuration is shown in Figure 2e and in more detail in Figure 3. The nanoprobe is rastered over the sample through an SPM feedback mechanism, usually in shear force [40] or tapping mode [41]. Shear force feedback is acquired by attaching a probe to one arm of a tuning fork [42]. Scattered photons are detected by correlating with phase and amplitude modulations of the tuning fork in SPM feedback. For SNOM experiments that utilize tapping mode with a cantilever based probe, scattered photons are detected by demodulating the signal with a lock-in at harmonics $(n)$ of the tapping frequency $(f)[43]$.

The experimental advancements described above utilized the nanoprobe as a scattering element only, and did not use the nanoprobe as an evanescent light source as proposed by Wessels. The use of a metallic probe to function as a confined optical field illumination source was first 


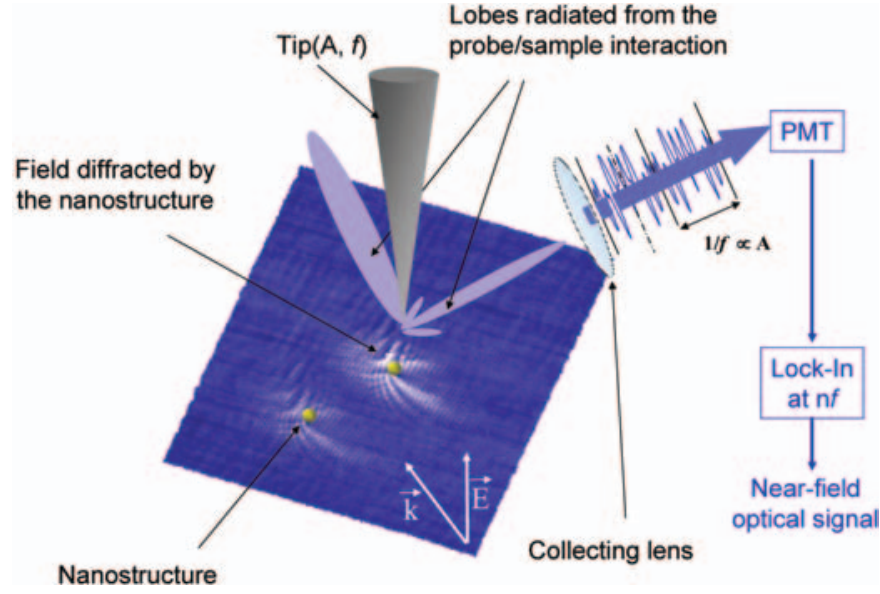

Fig. 3. The basic operation of apertureless SNOM is shown. Scattering lobes radiated from the probe/sample interaction region are collected in the far-field by the detection optics. In some configurations, such as tapping mode, a lock-in can be used to demodulate the signal for better signal to noise.

performed by Xie and colleagues (see Fig. 2f) [44]. This method has evolved through the careful generation of tips and the use of different illumination conditions. Particularly with the use of short pulse light sources, the generation of second harmonic fields and even white light is enabling apertureless SNOM greater utility in near-field measurements $[45,46]$. In addition to new, spatially resolved spectroscopies that are possible with this method, apertureless SNOM has arguably better spatial resolution than aperture SNOM due to the much smaller probe size.

\subsubsection{Photon scanning tunneling microscopy (PSTM)}

PSTM is a variation of aperture SNOM in the sense that it utilizes a tapered optical fiber and deserves specific mention due to its wide use. The illumination geometry is most closely given by the configuration shown in Figure 2d, whereby total internal reflection at the sample surface occurs. Typically, the probe is brought near a sample surface, and the fiber is rastered over the sample without feedback. A portion of the light that is scattered by the sample is collected by the fiber probe. In general, PSTM operates in a constant height mode, where the tip does not follow the topography. This method provides sub-diffraction limited optical information, but does not permit simultaneous topography to be collected [47-49]. However, it has an important advantage in that topography artifacts that can plague other SNOM studies are not possible in the constant height mode [50]. PSTM can also be invoked to include force feedback mechanisms, in which case STOM (scanning tunneling optical microscope) is another acronym frequently used [48]. A constant of this method, however, is the total internal reflection geometry.

\subsection{Metal nanoparticle preparation}

While a detailed discussion of metal nanoparticle preparation methods is beyond the scope of this review, a brief discussion of the types of samples that are typically probed with SNOM may be helpful to the reader. In most cases, the nanometric metallic samples probed with SNOM are made through colloidal synthesis or electron beam lithography. The most straightforward methods for colloidal synthesis utilize the reduction of metal salts, such as $\mathrm{AgNO}_{3}$ or $\mathrm{AuHCl}_{4}$ [51]. The reducing agent can strongly effect the size and homogeneity of the colloidal particles, with typical reducing agents being sodium borohydride or sodium citrate. When the $\mathrm{Ag}$ or $\mathrm{Au}$ ions are reduced to the neutral state, they do not remain soluble and seek a more stable solvated state by binding with another ion. The process of colloidal synthesis seeks to provide a balanced control of the aggregation process to produce similar sized colloids. The current state of colloidal synthesis is such that size distributions of $5 \%$ or less are reasonably common [52,53]. Also, by using surfactants that create micelles of different shapes, it is possible to synthesis elongated, metal nanorods or nanowires through colloidal synthesis. Other methods even produce prisms and other unusual shapes [22,54].

Electron beam lithography is used when one and two dimensional arrays of very closely spaced particles are desired. It is also used if a particular geometry between closely spaced nanoparticles is desired, or if a particular size and shape of nanoparticle is of interest. It is an inherently low throughput sample preparation method, but does produce high quality nanometric structures. The methods of this technique are well-developed, a nice reviews for the interested reader can be found in the literature [55].

\section{Single metal nanoparticle imaging}

Single metal nanoparticle imaging began with imaging defects in metal films and fractal materials. The first such report was that of Fischer and Pohl in 1989, which reported the imaging of gold nanoparticle defects in an otherwise flat film [56]. In this article, an ingenious set of probes was created by evaporating polystyrene spheres of $90 \mathrm{~nm}$ diameter onto a glass slide with a mean spacing of tens of microns. The probe was then coated with a gold film of $20 \mathrm{~nm}$ thickness. This was mounted on a piezoelectric stage and raster scanned, while illumination from the back of the probe in a total internal reflection geometry produced small radiating sources in which to probe a metal film. Since the spacing between probe elements was large, a microscope objective could straightforwardly collect the photons from a single probe-sample interaction. Using this configuration, a set of nanometric defects on the film was probed, and the scattered signal intensity and the full-width half-maximum (FWHM) as a function of probe height was monitored. Correlations with the expected dispersion curve for a metallic sphere on a metal film were made. 
Following this initial example, more conventional PSTM was used for monitoring nanometric defects. For example, Tsai and colleagues used PSTM in 1994 to monitor the near-field scattering from fractal materials. They observed strong spatial localization of energy in these systems, along with a strong dependence on the illumination polarization and wavelength [57]. The hot spots were thus assigned to localized surface plasmons, verifying the resonant optical theory of fractal materials. Similar observations were more recently reported by Gresillon et al. using an apertureless SNOM [58]. SNOM studies of random nanoparticle films have also been reported, where the films are the walls of optical waveguides [59].

The first example of PSTM images of isolated metal nanoparticles on a dielectric substrate was made by Krenn et al. in 1995 [60]. Two dimensional arrays of silver nanoparticles were made through electron beam lithography. In particular, arrays that had different sizes of nanoparticles with alternating $150 \mathrm{~nm}$ and $300 \mathrm{~nm}$ lengths along the major axis of an oblate spheroid shape were studied. The results showed that the illumination wavelength of $543 \mathrm{~nm}$ produced a strong near-field optical signal, while the longer particles did not show enhanced signal. It was proposed that the plasmon resonance of the larger particles was too far to the red to permit near-field enhancement. Silver island films were also prepared. Similar effects were seen in that some particles showed significant near-field signal while others did not. It was concluded that in both the arrays and the silver island films, there was a wide distribution of plasmon resonances that depended upon shape and size. As a result, only a fraction of the nanoparticles illustrated enhanced near-field optical signal.

The first reference to imaging metal nanoparticles through an illumination mode aperture SNOM method appeared in 1998 with the work of Klar and colleagues [24]. Shear force control was used to image gold nanoparticles with a particular motivation to resolve homogeneous plasmon linewidths through single nanoparticle detection for the first time. The homogeneous linewidths $(\Gamma)$ of plasmon resonances are extremely important to many of the applications of metal nanoparticles. For example, the success of coherent dipole-dipole coupling of plasmons in metal nanoparticle arrays, of interest for propagating photons with lateral widths well below conventional diffraction limits, relies on coherent interaction between nanoparticles $[17,18,61,62]$. Since the homogeneous linewidth of the plasmon resonance is inversely proportional to the dephasing time $T_{2}$, the success of such structures strongly relies on the smallest $\Gamma$ possible. Another important ramification of $\Gamma$ is on surface enhanced Raman scattering (SERS), in which the SERS cross-section is proportional to $\Gamma^{-4}[63]$.

For the particular case of metal nanoparticles, Klar et al. stated that single nanoparticle spectroscopy was advantageous for determining $\Gamma$ over the more typical method of hole burning. This is due to the hole burning requirement that $\Gamma$ be much less than the heterogeneous linewidth. However, with plasmon far-field spectroscopy of reasonably homogeneous sized particles, $\Gamma$ is only fractionally smaller than the heterogeneous linewidth. The particular sample used by Klar et al. was a $200 \mathrm{~nm}$ thick film of a $\mathrm{TiO}_{2}$ dielectric sol-gel matrix with a dilute concentration of $20 \mathrm{~nm}$ diameter gold nanoparticles. An $80 \mathrm{~nm}$ diameter aperture probe was used and maintained at a $7 \mathrm{~nm}$ height above the sample. The end result was that homogeneous linewidths of approximately $160 \mathrm{meV}$ $\left(T_{2}=8\right.$ fs) was found, as compared to $300 \mathrm{meV}$ for farfield spectroscopies of the film that averaged over many nanoparticles. The authors also found evidence for two interacting gold nanoparticles through a double peak in the SNOM transmission, with one peak at higher photon energies than the single nanoparticles. A model predicting the increased photon energy was developed for orientationally averaged samples. It is generally shown today that coupled nanoparticles have lower photon energy than single particles, although particular orientations of dimers relative to the incident light polarization can have higher energy resonances [64].

Also in 1998, the first report of apertureless SNOM methods to image metal nanoparticles appeared with the work of Nesbitt and colleagues [25]. In this work, a commercial Si cantilever AFM probe was used and operated in tapping mode. Approximately $14 \mathrm{~nm}$ diameter gold particles were dispersed on a prism and illuminated with an evanescent wave through total internal reflection. The primary conclusion of this work was that the interaction of the Si tip with the metal nanoparticle produced scattering that was orders of magnitude larger than that of the calculated scattering cross section of the nanoparticle alone, given quantitation of the evanescent field strength and the percent of scattered radiation collected by the far-field collection optics. The authors concluded that the Si tip was an effective antenna that amplified the nanoparticle scattering efficiency.

A second report by Adam et al. used apertureless SNOM with a cantilever probe to analyze the plasmon resonance energy of isolated gold nanoparticle colloids deposited on glass [65]. In this report, an $\mathrm{Ar}^{+}$laser was used to illuminate the $40 \mathrm{~nm}$ diameter gold particles in a total internal reflection geometry. The discrete laser lines of the $\mathrm{Ar}^{+}$laser were used to scan through the plasmon resonance energy from $457 \mathrm{~nm}$ to $529 \mathrm{~nm}$. The authors found that the plasmon energy peaked at $488 \mathrm{~nm}$ for $p$-polarized light, i.e. exciting the plasmon resonance perpendicular to the substrate plane. On the other hand, no resonance peak was found for $s$-polarized illumination that was polarized parallel to the substrate plane. Furthermore, the $488 \mathrm{~nm}$ peak was surprising in that it was higher in energy than estimated from the electric dipole approximation, which placed the resonance for a $40 \mathrm{~nm}$ diameter particle on quartz near $530 \mathrm{~nm}$. The authors explained these results by analyzing AFM images that showed the particle was actually deformed on the substrate, being approximately $60 \mathrm{~nm}$ wide and only $20 \mathrm{~nm}$ high. This was proposed to be due to either wetting of the metal nanoparticle on the quartz substrate or gentle tapping deformation from the tip. The $p$-polarized light excited the short vertical axis of 
the nanoparticle, producing a higher plasmon resonance energy as expected for smaller particles. The $s$-polarized light did not show a near-field enhanced signal because the $60 \mathrm{~nm}$ diameter width would have a resonance near $580 \mathrm{~nm}$ and would be beyond the reddest line of the $\mathrm{Ar}^{+}$ laser. The authors also pointed out that the $p$-polarized light may enhance the creation of confined light at the tip apex, similar that for metallic tips. This would strongly favor enhancement of the signal from the plasmon excited along the short axis of the nanoparticle.

In 2003, Wurtz et al. presented an apertureless nearfield optical study of the field diffracted by $\mathrm{Ag}$ and $\mathrm{Au}$ nanoparticles [66]. The work was performed with illumination from a $\mathrm{Kr}^{+}$laser in total internal reflection at $415 \mathrm{~nm}$, and thus was closer to the plasmon resonance of Ag particles than Au particles. A Si cantilever AFM probe was used. It was shown that the $\mathrm{Ag}$ and $\mathrm{Au}$ particles had significant differences in the near-field distribution of the normal component of the scattered field using $p$-polarized light (Transverse Magnetic, TM) illumination. A strong spatial confinement along with an enhancement of the normal component of the field were characteristic of the silver particles, while far-field contributions to the scattered field dominated for the gold particles (Fig. 4). The results were analyzed in terms of the illuminating field being in resonance only with the plasmon of silver particles, thus leading to enhanced near-field contrast for silver particles. In both cases, however, the incident field was found to be partially coupled into a far-field mode of identical polarization that scattered from the surface at an angle of $19^{\circ}$. Interestingly, this angle is very similar to theoretical and experimental studies of plasmon polaritons in metal films scattered at nanometric defects. The strong differences in the spatial profile of the scattered light depended strongly on illumination polarization. When illuminated with $s$-polarized light (Transverse Electric, TE), only confined signal was observed for both silver and gold. These results are important for the design of devices using metal nanoparticles to control electromagnetic energy transport, for example with plasmon polariton transport in metal nanoparticle arrays as it will be discussed in Section 5 [18].

\section{Advanced SNOM imaging methods}

In this section we describe several improvements in SNOM methodologies that enable efficient near-field investigations of metal nanoparticles.

\subsection{High harmonic demodulation in apertureless SNOM}

New techniques have enabled improved spatial resolution for imaging the near field of metal nanoparticles. One of the most successful is the demodulation of scattered signal at higher harmonics than the fundamental of the cantilever tapping frequency. A schematic of the concept behind this technique is shown in Figure 5. This method allows the experimentalist to preferentially detect (a)

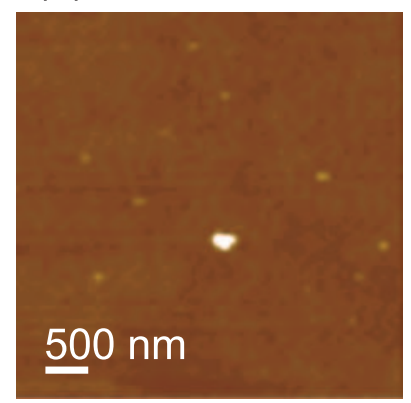

(c)

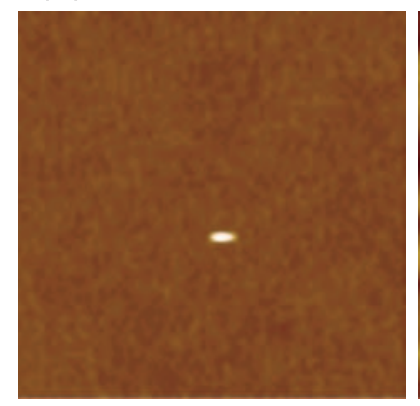

(b)

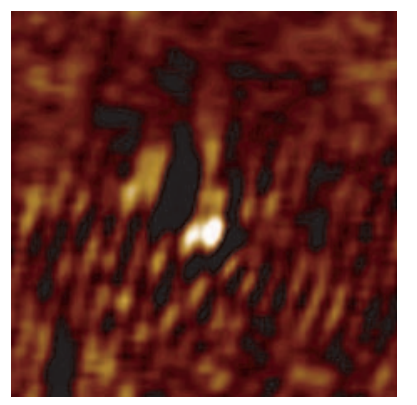

(d)

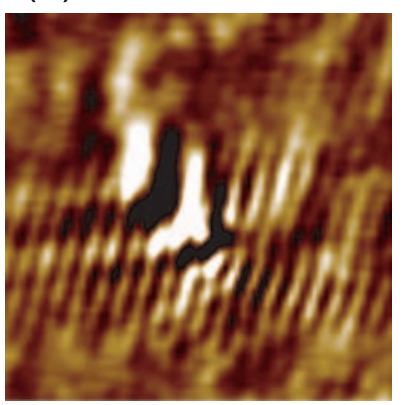

Fig. 4. Metallic nanoparticles illuminated in total internal reflection at $415.4 \mathrm{~nm}$ on a glass substrate. (a) and (b) correspond to the respective AFM and SNOM contrasts of a $40 \mathrm{~nm}$ diameter Ag particle, while (c) and (d) correspond to the respective AFM and SNOM contrast of a $25 \mathrm{~nm}$ diameter Au particle. The illumination polarization is TM and the wavevector points from bottom right to top left. SNOM images represent the normal component of the scattered field.

the fields of high longitudinal gradient (i.e. perpendicular to the surface). The optical response of a scatterer vibrating sinusoidally (frequency $f$ ) in this gradient is nonlinear, leading to high harmonics in the scattered light $(2 f, 3 f$, $4 f, \ldots)$. As described by Hillenbrand and Keilmann, the result is that the $n$th order Fourier component will exceed that of the background field due to the nonlinear nearfield interaction [67]. Thus, the demodulation of the scattered light at lower harmonics favors fields with significant amplitude over the cantilever vibrational amplitude range (such as propagating waves). As higher harmonics of the field are used, successively smaller regions over the period of the cantilever motion are selected, with particular emphasis on the regions of tip-sample interaction. This favors more confined fields nearer the surface of the sample (especially evanescent fields). This was first demonstrated by Wurtz et al. in 1999 for characterizing the spatial profile of stimulated emission from laser diodes [43], and then applied to metal nanoparticle structures by Hillenbrand and Keilmann in 2000 [67]. A consequence of this method is that one must be careful with interpreting the spatial profile of detected fields, simply because the fields will be nonlinear with $z$ except for small cantilever vibrational amplitudes. 

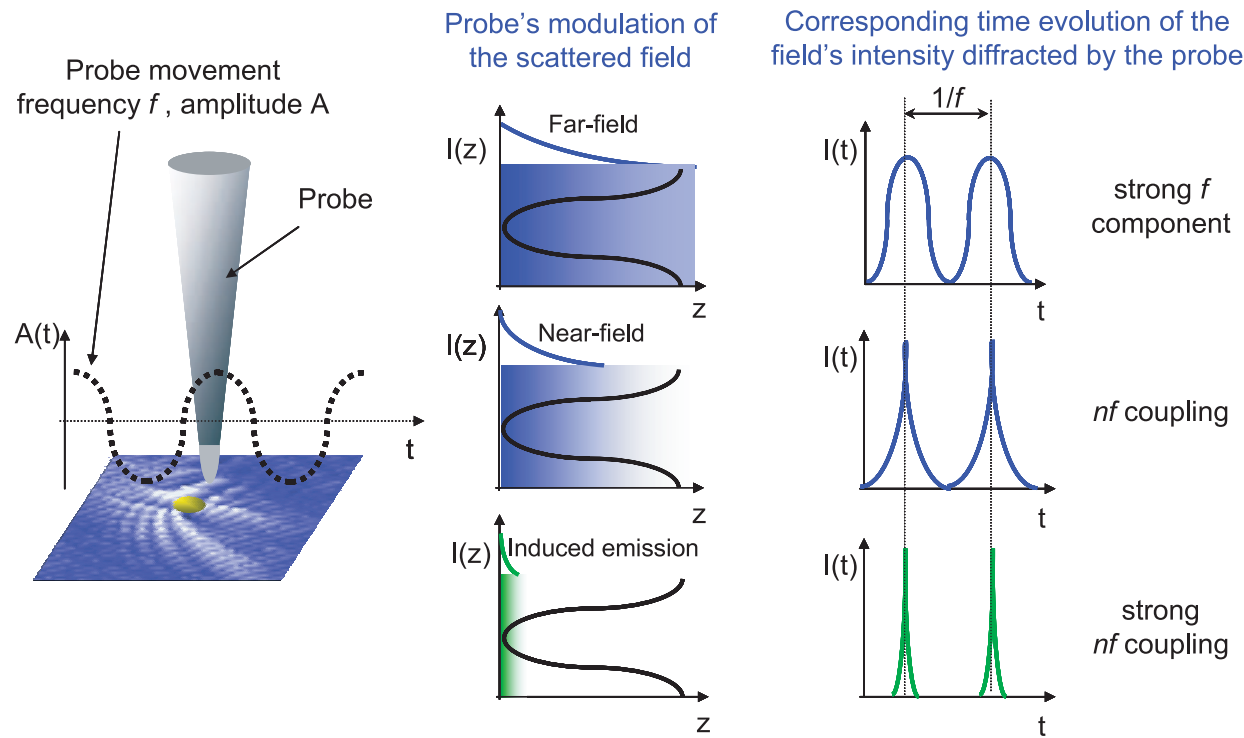

Fig. 5. The principle of higher harmonic detection for tapping mode apertureless SNOM is shown.

\subsection{Apertureless heterodyne detection}

The above research describes efforts to resolve the intensity of the optical near-field of isolated metal nanoparticles. However, a full description of the nanoscale optical processes would ideally provide both phase and amplitude information for evanescent fields in nanostructures. Such information could provide important information, for example, of the phase of plasmonic responses as a function of nanostructure shape, size, environment, and near-field coupling.

With this thought in mind, arguably the most important development for apertureless SNOM studies in recent years has been the development by Hillenbrand and Keilmann of heterodyned apertureless SNOM at visible wavelengths $[67,68]$. The concept is straightforward, in that an interferometer is created in which the signal arm consists of the light collected from the scattering from the apertureless SNOM probe $\left(E_{S}=\left|E_{S}\right| \exp \left[-i\left(\omega t+\phi_{s c}\right)\right]\right)$, and the reference arm $\left(E_{R}=\left|E_{R}\right| \exp [-i(\omega t+(\Delta \omega t+\right.$ $\left.\left.\left.\phi_{R e f}\right)\right]\right)$ is subsequently overlapped with it. Here the reference arm is frequency shifted by $\Delta \omega$ from that of the signal arm with the use of an acousto-optic modulator in the reference arm. In this way, the collinear reference and signal beams will create a temporally modulated interference that can be demodulated with a lock-in. For a frequency shift $\Delta \omega$, the scattered signal in heterodyne detection will then be proportional to $\left|E_{S}\right|^{2}+\left|E_{R}\right|^{2}+$ $\left|2 E_{S} E_{R}\right| \cos \left(\Delta \omega t+\phi_{R}-\phi_{S}\right)$. From this relation, three important factors are clearly manifest in the new cross term produced as a result of the interferometric detection. First, amplification of the scattered signal by several orders of magnitude is expected because $E_{R}$ is much larger than $E_{S}$. Second, the phase difference between the reference and signal arms can be detected as a result of the time varying interferogram, if the signal is demodulated at the tapping frequency $(f)$ minus $\Delta f$ or $n f-\Delta f$, where $n$ is an integer and $\Delta f=\Delta \omega / 2 \pi)$. Third, it can be used to elim- inate signal from unwanted interferences in apertureless SNOM. Such effects include optical interference patterns created between the scattered field and the background evanescent illumination field $\left(E_{b}\right)$ present on the sample surface. This interference effect has been an issue that has long plagued apertureless SNOM [69], but heterodyne detection permits one to control this effect. This is possible because the $E_{b} \times E_{s}$ cross term is modulated only at $n f$, and not $n f-\Delta f$, so that lock-in detection at $n f-\Delta \mathrm{f}$ removes the unwanted cross term.

Heterodyne detection has already produced significant advances in SNOM for metal nanoparticles. The first report of this nature was by Hillenbrand and Keilmann in 2001 [70]. In this work, a sample of Au nanoparticle aggregates with wide size dispersion was probed with a HeNe laser output at $633 \mathrm{~nm}$ in a heterodyne SNOM configuration. With this sample and detection configuration, it was possible to observe single particles within the aggregates that showed strong enhancement of the near-field response. The particles that showed enhancement were neither the lowest or highest particles in the SNOM scan, thus eliminating artifacts as the enhancement mechanism. Rather, only a few particles in the aggregates had the size or shape to have a plasmon resonance very near the $633 \mathrm{~nm}$ illumination wavelength. Since the phase and amplitude of the SNOM measurements of the single particles were uniform over the spatial extent of the particle, this was used to show that the observed response was due to an oscillating dipole in the $z$ direction (the direction perpendicular to the substrate). Other aggregate structures, however, showed nonuniform phase distributions, even $180^{\circ}$ shifts in the phase of the near-field response. The results in these cases were assigned to the detection of quadrupolar modes. Finally, this publication reported the observation of "gap" modes of closely spaced metal nanoparticle structures. As discussed above, closely spaced metal nanoparticles can have greatly enhanced 
optical fields in between the particles, as the enhanced field of one particle is then enhanced a second time upon interaction with the second nanoparticle [20]. The results are shown in Figure 6. For this measurement, Si tips were used because they are sharper than the PtIr tips used for the prior measurements. As Figure 6 shows, although some of the individual particles show enhanced near-field intensity, by far the largest enhancements are between the particles. This is even more remarkable in that Figure $6 \mathrm{~d}$ shows that the probe is still too large to reach the regions of largest enhancements that are lower in the sample than the probe can reach.

Following this initial work, a second study by Hillenbrand and Keilmann sought to achieve higher spatial resolution of metal nanoparticle near-fields by using a carbon nanotube (CNT) bundle attached to the tip of conventional cantilever Si probes [71]. The $25 \mathrm{~nm}$ radius CNTs, while broader than the apex of a $\mathrm{Si}$ probe, do not broaden above the apex as conventional conical probes do. This is an important direction to pursue, since the evanescent fields of surfaces are scattered to some extent by the conical probe when the typical exponential decay lengths of $\sim 150 \mathrm{~nm}$ are approached. The sample in this case was created through electron beam lithography, which was an array of $\mathrm{Au}$ nanoparticle disks, $20 \mathrm{~nm}$ high and 90 to $140 \mathrm{~nm}$ in diameter. As Figure 7 shows, the sample was illuminated in a geometry in which the oscillating dipole of the metal nanoparticle is parallel to the substrate rather than perpendicular. As shown in Figure 7a, this should produce a near field response that has a different phase at the opposite ends of the particle. Simulations for this effect are shown in Figure $7 \mathrm{~b}$. The experimental data shown in Figures $7 \mathrm{c}$ and $7 \mathrm{~d}$ bear out that the phase is opposite at each end of the particle. It should be emphasized that this observation was enabled by the unique capability of the heterodyne version of the apertureless SNOM. Although the CNT bundles enabled this study, the spatial resolution of this method should continue to improve dramatically as an isolated, single, single walled carbon nanotube is employed.

\subsection{Phase sensitive imaging using aperture SNOM}

Aperture SNOM methods have also been used to extract phase sensitive images of metal nanoparticles. Prikulis and colleagues recently used aperture SNOM in a geometry that enabled observation of the interference between the illuminating field from the aperture and that of the scattered field of the nanoparticle [72]. Their theoretical modeling showed that a zero phase shift should be observed for plasmon resonances of higher energy than the illuminating field, which should then shift by $\pi$ as the illuminating field is higher in energy than the plasmon resonance. Experimental studies of $\mathrm{Ag}$ nanoparticles demonstrated this effect, but only for the case where the photon energy was lower than the plasmon resonance.

Similar experiments on single metal nanoparticles and nanostructures were performed by Klimov and colleagues, this time using a white light spectral continuum created by ultrashort femtosecond pulses from a Ti:sapphire oscillator [73]. Their results clearly support the concept of constructive interference between the illuminating field and scattered field for photons of energy lower than the plasmon resonance and destructive interference for photons of higher energy than the plasmon resonance. The white light continuum allows for precise determination of the plasmon resonance at the crossover point from constructive to destructive interference. They have applied this technique to more complex structures such metal nanoparticle dimers bound by porphyrin linkers, and found that they can detect small changes in dyad structure or size inhomogeneities with this method.

\subsection{Photoluminescence imaging of metal nanoparticles}

Mooradian first reported the phenomenon of a broad photoluminescent response from metals when photoexcited with the CW output from an $\mathrm{Ar}^{+}$laser [74]. The advent of pulsed lasers more easily produced nonlinear optical processes in metal nanoparticles that could conceivably be used as a contrast mechanism for imaging the near-field of the particles. In 2003, Bouhelier et al. used a femtosecond laser to produce photoluminescence from a $\mathrm{Au}$ rod on a glass substrate, and subsequently to collect the photoluminescence in a SNOM geometry [75]. The fs laser illumination permitted two photon absorption, so that the excitation at $780 \mathrm{~nm}$ was well separated from the observed photoluminescence peaking at $650 \mathrm{~nm}$. The AFM topography and photoluminescent SNOM images are shown in Figure 8. In this case, the polarization of the involved field is parallel to the long axis of the rod. This permitted the Au nanoprobe, approximately $10 \mathrm{~nm}$ in diameter, to function solely as a passive scattering element because the light was not polarized along the long axis of probe, thereby avoiding field enhancement effects of the probe. This also enabled the $\mathrm{Au}$ nanoprobe to function without perturbing the spectral profile of the photoluminescence. This was shown by observing the photoluminescence through confocal microscopy in the absence of a probe, and the identical emission spectrum was found.

\section{Near-field imaging of arrays of metal nanoparticles}

Arrays of metal nanoparticles are of significant technological and scientific interest because inter-particle coupling can produce collective effects that alter plasmon resonance energies, damping, and near-field spatial profiles. Since efforts are beginning that seek to understand the near-field optical processes of nanoparticle arrays, an understanding of the far-field characterization efforts that have been undertaken is necessary. The coupling in periodic arrays can take two different forms: radiative coupling or nearfield coupling. Radiative, or dipolar, coupling is the primary mechanism of collective scattering when particles are spaced at distances large compared to the size of the particles. This form of coupling is due to far-field interference 
a

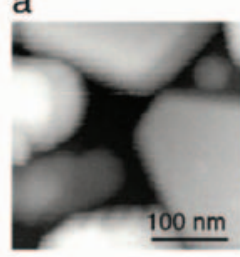

b

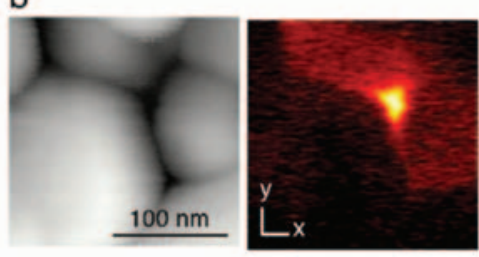

C

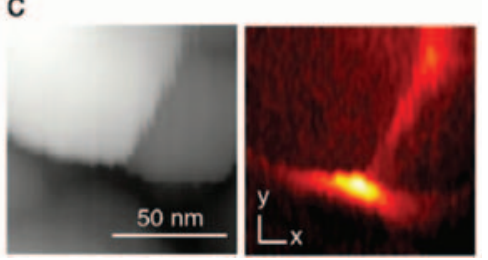

d

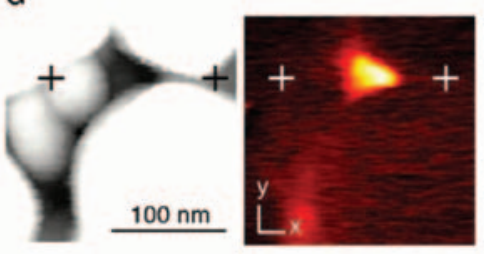

f

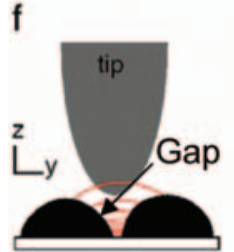

e

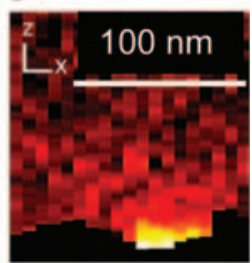

Fig. 6. The topography (left) and near-field amplitude (right) for gap modes between closely packed Au nanoparticles are shown in (a)-(d). Figure (e) is the result of a $x-z$ SNOM scan that reveals the high $z$ confinement of the gap mode, and (f) illustrates the need for narrower probes to access confined fields in such nanostructures. Figure provided courtesy of R. Hillenbrand.

(a)

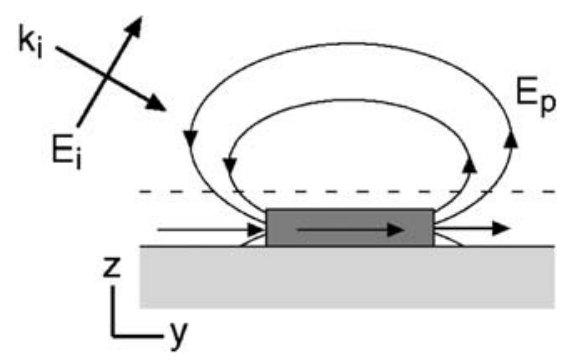

(b)

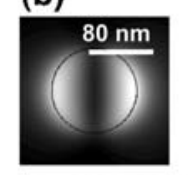

(c)

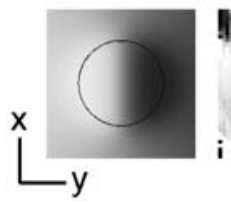

(d)

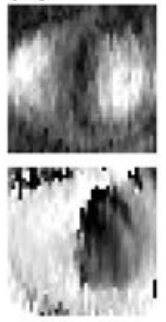

Fig. 7. (a) A schematic of the illumination geometry for the phase sensitive SNOM studies of Hillenbrand and Keilmann is shown. (b) is a theory image of the intensity and phase of the field in the $z$ direction, $10 \mathrm{~nm}$ above the sample, for the Au disks used. (c) and (d) illustrate typical experimental results and show the $180^{\circ}$ phase shift of the optical near field across the Au nanoparticle. Figure provided courtesy of R. Hillenbrand.
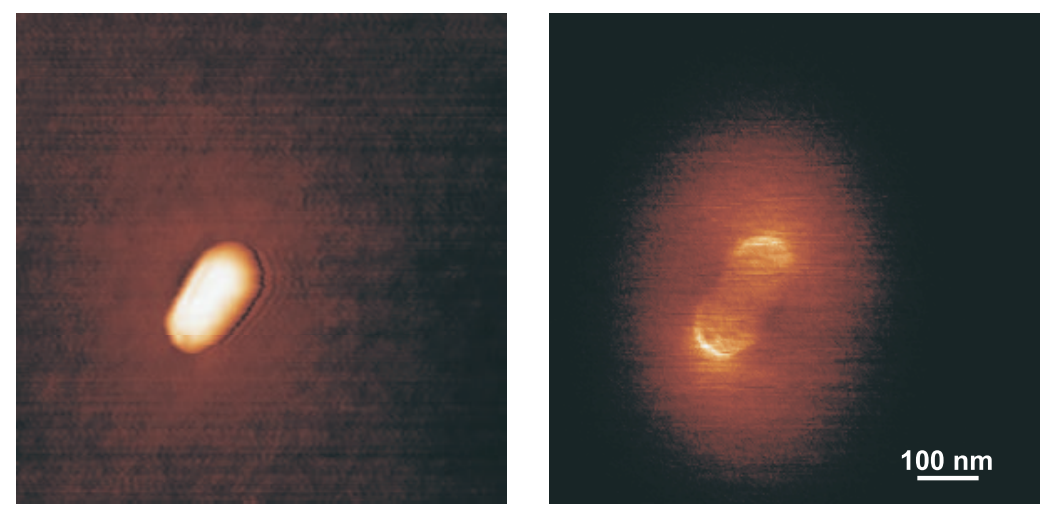

Fig. 8. The AFM (top) and SNOM (bottom) image of the photoluminescence from a Au nanorod is shown. Figure provided courtesy of A. Bouhelier. 
between the scattered light fields of individual particles to give collective plasmon behavior that is different than the isolated particles. On the otherhand, near-field coupling between particles dominates for particles with smaller spacings where each particle lies within the evanescent field of neighboring particles. The theory and experiment of collective interactions of metal nanoparticle arrays in the dipolar regime are well-developed. In 1984, a theoretical study by Meier et al. showed that there is an interplay between resonance shifts and damping of the plasmon [76]. As a grating spacing is increased and approaches a grating order that induces light fields to change from evanescent to radiative, there is a red-shift of the plasmon resonance for perpendicular incidence. At the spacing where a grating order becomes radiative, a strong damping of the plasmon occurs while the plasmon resonance now blueshifts with increasing lattice spacing. This was beautifully shown in a far-field experimental study by Lamprecht et al. for gold nanoparticles with grating constants from $350 \mathrm{~nm}$ to $850 \mathrm{~nm}$ [77]. Another study by Haynes et al. was performed on arrays of both square and hexagonal symmetry, using nanoparticles of different shapes as well [54]. They also observed a strong red shift in the plasmon resonance as the lattice constant increased. They proposed that at lattice constants below $100 \mathrm{~nm}$, the plasmon should blue shift as the spacing increases, due to near-field coupling. However, lithographic limitations prevented exploration of this region. They did cite red shifting of the plasmon resonance in three dimensional arrays of gold nanoparticles that were created through self-assembly [78]. A two dimensional array of $\mathrm{Au}$ nanoparticles created through scanning probe lithography was also reported by Kim et al. [79]. SNOM measurements for particle spacings greater than 1 micron were reported.

A two dimensional array structure with potential nearfield interactions was designed and studied by Rechberger et al. [64]. In this study, pairs of nanoparticles were arranged into a two dimensional lattice structure. The pairs consisted of $150 \mathrm{~nm}$ diameter particles that were spaced by varying distances of $150 \mathrm{~nm}$ (near touching) to $450 \mathrm{~nm}$ center-to-center. Note that the larger lattice spacing is in the regime where dipolar coupling should dominate. As the spacing is decreased, the plasmon resonance redshifts significantly for light polarized parallel to the particle pair, in contrast to the blueshift that occurs for dipolar coupling. This indicates that nearest neighbor coupling dominates for the pairs, in agreement with the isolated particle pair studies of Tamaru [80].

A related study was undertaken by $\mathrm{Su}$ and colleagues, whereby a dark field scattering configuration was used to study individual pairs of elliptical gold nanoparticles created through e-beam lithography [81]. The experimental geometry was optimized to produce rather extraordinary signal to noise for the individual pairs of particles. A redshift of the plasmon resonance was observed for illumination polarization parallel to the long dimer axis (which is parallel to the short axis of the individual elliptical particles). A redshift was observed beginning with a centerto-center spacing of $\sim 250 \mathrm{~nm}$, when the short axis was
$84 \mathrm{~nm}$ and the long to short axis ratio of the particles was 1.55. For the closest spacing of $138 \mathrm{~nm}$ center-to-center, a redshift of approximately $60 \mathrm{~nm}$ occurs relative to the individual particle. Interestingly, it was noted that the $\mathrm{Cr}$ layer typically deposited as an adhesion layer on the substrate before the $\mathrm{Au}$ is deposited produces significant additional damping of the plasmon. Such an observation is important for the functioning of plasmonic materials.

Despite the very interesting results of far-field imaging of two dimensional arrays of metal nanoparticles, there have been no studies to the author's knowledge of SNOM studies of two dimensional arrays with short spacings of the order described above. There have also been SNOM studies on two dimensional arrays where the spacing was much larger [82]. This work was a detailed study on the effect of wavelength, polarization, and angle of incidence on the calculated and observed near-field image. Efforts to observe and predict the interference patterns for farfield coupling between light scattered from the individual nanoparticles was reported. Ultimately, closer spaced metal nanoparticle arrays would be useful for SNOM studies. Since the resonance and damping of the plasmon is significantly modified in the arrays, it is likely that significant modulation of the near-field spatial profiles would be altered and measurable through SNOM studies.

Contrary to closely spaced, two dimensional arrays, one dimensional arrays of closely spaced nanoparticles have been studied in detail with various SNOM techniques. As mentioned in the introduction, this is largely due to the interest in the ability to directionally delocalize the surface plasmon of metal nanoparticles. If propagation is possible, it would mean that electromagnetic energy could move in a pseudo-waveguide environment with lateral widths far below the diffraction limit of dielectric waveguides. Such an idea was originally proposed for a linear chain of silver nanoparticles by Quinten et al., which built upon work by Takahara et al. for light propagation in nanoscale metal cylinders $[83,84]$. In the work by Quinten et al., it was proposed that a chain with $25 \mathrm{~nm}$ radius silver particles, spaced center-to-center by $75 \mathrm{~nm}$, would propagate the surface plasmon nearly 1 micron. For larger spacing, the damping increased rapidly as the nearfield coupling decreased. While at first glance the propagation lengths seem relatively short, applications that require such small lateral widths would generally be applied to nanoscale or micronic structures, thus reducing the need for long propagation distances.

In 1999, seminal research by Krenn et al. produced PSTM images of a chain of gold nanoparticles on a glass substrate [16]. The sample was created through electron beam lithography and consisted of a one dimensional array of particles with $100 \mathrm{~nm} \times 100 \mathrm{~nm}$ square dimensions and a $40 \mathrm{~nm}$ height. The center-to-center distance was $200 \mathrm{~nm}$. The results were compared to single particles of identical dimensions. The PSTM signals for single particles vs. a chain of particles were very different, with single particles having a spatial extent of the scattered field on the micron length scale, while the chains showed highly confined near-field optical signals of spatial extent on the 


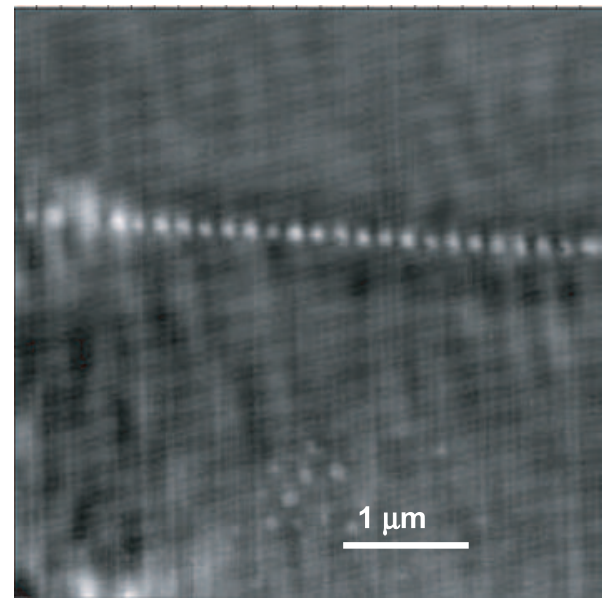

Fig. 9. An experimental PSTM image of a Au nanoparticle array is shown. Figure provided courtesy of J. Krenn.
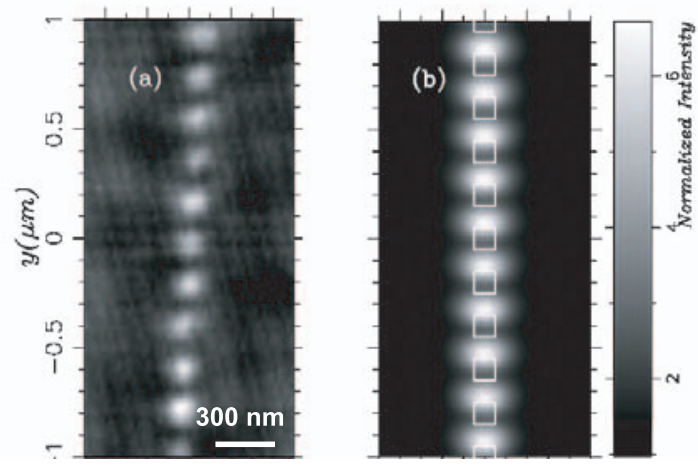

Fig. 10. Comparison between PSTM experiment (a) and theory (b) for the squeezed plasmon of Au nanoparticle arrays is shown. Figure provided courtesy of J. Krenn.

order of $100 \mathrm{~nm}$ (Fig. 9). The quantum involved in the confinement of the optical field was termed the "squeezed plasmon". Also very interesting was the location of the field relative to the array topography. By using theoretical modeling with a Green's dyadic technique, it was proposed that the fields are likely displaced between the particles, i.e. with the near-field maximum not on the particle but in between the particles as a result of the near-field interaction between the particles. The experiment and theory matched very well concerning the spatial extent of the field (Fig. 10). However, since PSTM was performed in the constant height mode and not sensitive to topography, it was not specifically experimentally verified that the optical near-field was displaced from the particles.

Since this initial discovery, Atwater and colleagues have advanced the concept that these arrays may function well as plasmon polariton waveguides of extraordinarily small lateral dimensions [18]. Their experimental efforts have been critical for understanding the types of structures and materials that are necessary to observe significant propagation lengths of the plasmon polariton. Early studies focused on Au nanoparticle arrays made through electron beam lithography, with particle diameters of
$50 \mathrm{~nm}$ and center-to-center distances of $75 \mathrm{~nm}$ [61]. In order to obtain significant extinction for ground state absorption spectra, several thousand 1D arrays were placed on 100 micron square grid and spaced by 1 micron. Since the radiative coupling depends on $d^{-1}$ and near-field coupling on $d^{-3}$, it was expected that near-field coupling would dominate the spectral data. Through white light illumination of the sample with a wavevector perpendicular to the substrate and polarized parallel or perpendicular to the long axis of the arrays, the longitudinal and transverse plasmon-polariton spectra were obtained. Strong evidence for collective interactions was obtained through the ground state absorption measurements because a significant peak splitting in the energy of the transverse and longitudinal modes was observed. The longitudinal mode was at lower energy $(2.06 \mathrm{eV})$ as predicted, while the transverse mode peaked at $2.12 \mathrm{eV}$. The peak splitting enabled a specific calculation of the interaction strength between particles as well as the bandwidth of the interaction that could support plasmon polariton propagation. It was found that the damping was too high, i.e. $3 \mathrm{~dB} / 15 \mathrm{~nm}$, to support this particular array design for plasmon polariton propagation. The authors, however, proposed that by using Ag for lower damping, and the use of rod shaped particles to increase coupling strengths, dramatic improvement in the performance of the arrays would result. The rods also have dramatically lower damping losses because the electron mean free path becomes less than the length of the long axis of the nanorods.

This type of structure was constructed and reported by the Atwater group in 2003, and SNOM played an integral role in the first demonstration that plasmon polariton propagation can occur in metal arrays [17]. Ag nanorods with a $3: 1$ aspect ratio were used. Specifically, $50 \mathrm{~nm}$ surface-to-surface distances and particle sizes of $90 \mathrm{~nm} \times 30 \mathrm{~nm} \times 30 \mathrm{~nm}$ were used. To this, fluorescent nanospheres were randomly distributed that absorbed photons in the spectral region of the plasmon resonance of the arrays in the mid-500 $\mathrm{nm}$ spectral region. A SNOM probe was rastered over the sample and the emission from the fluorescent nanospheres was collected in the far-field. The spatial profile of the fluorescence was monitored and compared to fluorescent nanospheres that were not on an array. The authors found that the spatial profile of the fluorescence was broader when on the arrays, consistent with the concept that if the array can propagate plasmon polaritons, the energy to excite the fluorescent nanoparticle can come from further distances than for nanospheres not on the arrays. The increase in width was found to be consistent with the measured nanoparticle coupling strength as judged by ground state absorption spectra of the arrays, which is on the order of $200 \mathrm{~nm}$. Thus, significant improvement over the $\mathrm{Au}$ nanoparticle systems was observed.

Others have also pursued the near-field optical characterization of one dimensional metal nanoparticle arrays. In 2003, Wurz et al. used tapping mode apertureless SNOM in total internal reflection geometry to study plasmonic arrays that consist of closely spaced Ag metal 
nanoparticles [62]. Confined optical signals within the array were observed along with a reduction in the far-field scattered signal (Fig. 11). Simultaneous collection of the atomic force microscopy signal and near-field signals also showed for the first time experimentally that the spatial distribution of the near-field is strongly modified in the arrays compared to isolated metal nanoparticles. The peak of the near-field intensity was displaced towards the gaps in the array, but was not centered and was dependent upon the illumination wavevector. A strongly decreased forward scattered angle (with chain length) relative to the substrate as compared to single metal nanoparticles was reported, as well as diminished overall forward scattering. Also, some regions of the arrays did not show significant near-field enhancement. This may be due to the irregular shape or size of a particle that brings that particular nanoparticle out of resonance with the rest of the array. It is certain that the ultimate success of this field will depend strongly on high quality samples that push the current limits on nanoparticle spacing, size and shape homogeneity of nanoparticles in closely spaced arrays.

\section{Metal tips for illumination mode imaging through tip field enhancement}

The use of metal probes in apertureless SNOM opens the possibility that the probe produces an enhanced optical field near the probe apex. This is due to the high density of charges at the apex that interact with the illumination field to create enhanced evanescent fields confined near the probe [85]. As a result, this opens an entirely new dimension to SNOM, in that spectroscopies can be performed on nanostructures with advantages over conventional aperture-based methods. For example, the size of the apex of an apertureless probe is smaller than current aperture probes by up to an order of magnitude. Second, the apex of an apertureless probe consists of one material, and not the metal coated dielectric materials that are typically used for aperture probes. Modeling tip-sample interactions is more achievable under these conditions than with an aperture probe. Under appropriate illumination conditions, it has been proposed that intensity enhancement factors of several thousand are possible at the apex of a metal tip [86,87]. Xie and colleagues were the first group to report the use of metal tips that specifically utilized the evanescent components of the enhanced field in an apertureless configuration [44]. In this work, the authors optimized the illumination geometry and tip shape to produce the enhanced field. Focused ion beam (FIB) milling was used to create the asymmetric probe shape that was predicted by electromagnetic calculations to obtain an incident polarization parallel to the tip axis (the necessary condition to excite an electromagnetic singularity). Also integral to the enhancement is the type of laser illumination used. In this case, femtosecond pulses at $800 \mathrm{~nm}$ from a Ti:sapphire oscillator were used, with peak powers important to induce high field enhancement. In this study, near-field images of J-aggregates and algae samples were collected, verifying the versatility of this technique. The authors pointed out that the apertureless method is well suited to biological samples, since tip heating in aperture probes can damage delicate samples.

Novotny and colleagues later reported elegant new capabilities of field enhancements at metal SNOM probes. They found that both confined second harmonic generation [88] and white light could be generated at the metal tip apex [46]. Thus, resonances can be probed that are not near the illumination laser wavelength, or transient absorption or scattering spectroscopies can be performed with the white light continuum at high spatial resolution. The ability to perform transient pump-probe spectroscopies at nanoscale dimensions would be a very powerful new capability for SNOM indeed.

\section{Theory}

Following the technical developments of SPM that ultimately enabled the collection of sub-diffraction limited optical information, theoretical pursuits have been among the most critical for enabling scientific conclusions from the acquired images. Theory has also been instrumental in guiding the future efforts of experimentalists. We briefly review recent theoretical developments here, but a complete review is beyond the scope of this article.

The theoretical methods, metallic nanoparticle structures, and experimental geometries addressed by theoreticians vary widely. Recently, Richard made important strides towards understanding the images of isolated metal nanoparticles on a glass substrate in a PSTM geometry by using a Green's dyadic technique $[89,90]$. He showed the expected spatial profiles of the individual components $\left(E_{x}, E_{y}, E_{z}\right.$, and $\left.E_{\text {Total }}\right)$ of the metal nanoparticle near-fields under total internal reflection illumination. The changes in the field components under different illumination polarizations, as well as the spatial profile changes for different materials and wavelengths were carefully described. Particular attention was paid to the role of the plasmon resonance and the ability to distinguish different metals based on their spatial profiles in the near-field. This work is critical for understanding near-field interactions between nanoparticles, where the near-field spatial profile under a particular illumination condition must be known to predict the particle-particle interactions. Martin and colleagues also used the Green's dyadic technique to model strongly localized fields in plasmon resonant nanorods with triangular cross sections [26,91]. In addition to localized hot spots, a broader plasmonic spectral response was found.

Another theoretical method is the multiple multipole method, which uses a series expansion of analytical solutions of Maxwell's equations [92]. The solutions have a free parameter that is found for the particular boundary conditions of a specific structure and illumination geometry [93]. This method is advantageous computationally because only the boundaries need to be discretized, while within the nanostructure an exact solution exists. This 

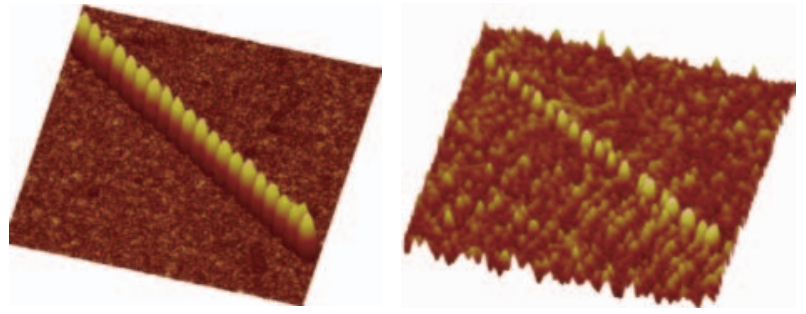

Fig. 11. AFM image (left) shows the topography of a linear array of $100 \mathrm{~nm}$ diameter silver nanoparticles, spaced by $200 \mathrm{~nm}$ center-to-center. The tapping mode SNOM image (right), collected simultaneously with the AFM image, illustrates the confined optical fields near the nanoparticles.

method has been used very successfully for near-field optical calculations by Novotny and colleagues for a variety of studies, particularly related to field enhancements near the apex of metal tips $[44,86]$. Other important contributions to scattering SNOM have been made by others, including Greffet and colleagues who used reciprocity theorems in electromagnetism to obtain an exact solution for SNOM signal with a variety of SNOM probes $[94,95]$. Specific applications for apertureless SNOM were described that addressed the dependence of SNOM signal on illumination polarization and wavelength. Pack et al. also explored signal formation in apertureless SNOM experiments using aggregate Mie theory [96]. In other works, Stockman and colleagues have proposed a variety of unique structures for nanophotonics applications [97,98].

The finite-difference time-domain (FDTD) method is a computationally efficient way to calculate light scattering and propagation in optical materials, and operates by solving Maxwell's equations for discrete time and space elements [99]. A variety of researchers have used FDTD for understanding near-field imaging and processes in metal nanoparticle structures. Atwater and colleagues have used FDTD for modeling the time dependence of the optical electric field created through collective plasmon excitation of one dimensional nanoparticle arrays [61]. Gray and colleagues have used the method to propose novel metal nanoparticle structures as well as correlate their predictions with SNOM experiments $[62,100]$. They used their methods to calculate the scattering of a single nanoparticle and chains of nanoparticles under evanescent optical excitation. An example of their results is shown in Figure 12 , where the total internal reflection of a plane wave is plotted as a function of time in the presence and absence of a $\mathrm{Ag}$ particle illuminated near the plasmon resonance. The scattering angle from the substrate into the far field shown was confirmed with SNOM experiments [62]. As supported by the experimental measurements, the scattering angle relative to the substrate was reduced dramatically for arrays of silver nanoparticles, thus providing support for chains of particles functioning as nanoscale, planarized optical elements.

As a final beautiful example of the power of theoretical efforts for interpreting SNOM images, Figure 13 illustrates recent results by Chicanne et al. on the near-field signals from corrals of metal nanoparticles [101-103]. In

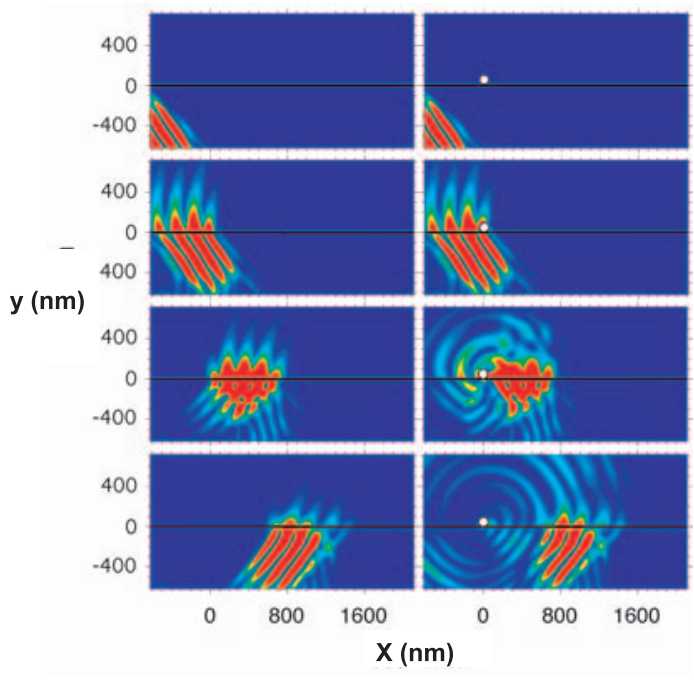

Fig. 12. Shown are the time sequences of the magnitude of the electric field from FDTD simulations. In all panels, refractive index $n=1$ for $y$ values above zero and $n=1.5$ for $y$ values below zero and a solid line is shown to mark the interface. The four panels on the left show a pulse of light emanating from the lower left quadrant of the $n=1.5$ medium, traveling up towards the interface at $y=0$, and undergoing internal reflection back into the medium. The time interval between panels is about $2.6 \mathrm{fs}$. The four panels on the right correspond to the same situation except now a $100 \mathrm{~nm}$ diameter $\mathrm{Ag}$ nanowire has been placed on top of the $n=1.5$ medium near the origin. In order to see the scattered light clearly, we use a color scale such that blue is zero field and red corresponds to field magnitudes greater than or equal to $0.1 E_{o}$, with $E_{o}$ equal to the incident maximum strength. Figure courtesy of S. Gray.

the particular SNOM geometry utilized, it was found that the near-field patterns were in agreement with theoretical calculations of the spatial distribution of the optical local density of states (LDOS) of the corral. The ability to produce and probe patterned LDOS should enable the observation of novel physical effects, such as modulation of molecular emission quantum yields or new materials for photonic band gap structures [104]. The authors point out that LDOS is specific to a given nanostructure, regardless of the process of signal formation via the nanoprobe. The ability to observe the LDOS showed that the probe was purely passive in the signal collection (Fig. 13). The experimental efforts employed a collection geometry SNOM configuration in constant height mode to obtain the images shown in Figure 13 [101].

\section{Conclusions}

Nanoscale metallic structures have an extraordinary future in optics, electronics, and sensing technologies. However, an appreciation for the importance of high spatial resolution in order to study and harness the optical nearfield of metal nanoparticles is very recent. The works described above are likely the initial glimmerings of a brilliant future for the near-field optical properties of metal 

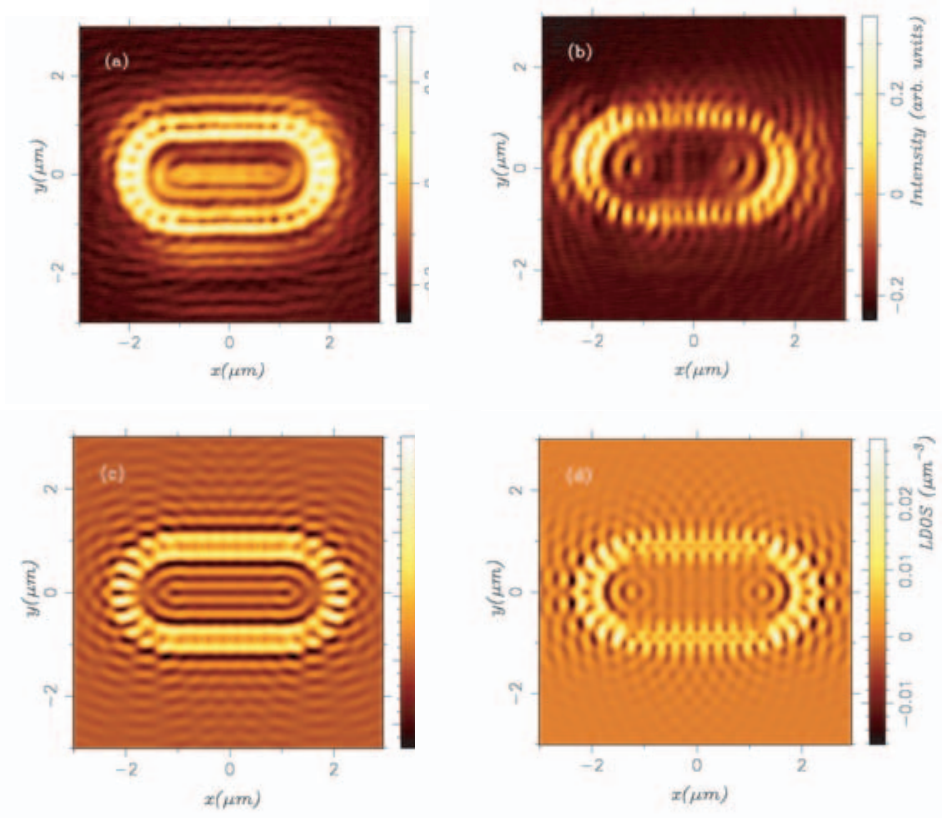

Fig. 13. Experimental SNOM images (top) and theoretical LDOS changes (bottom) are shown for a corral of Au nanoparticles that are $100 \mathrm{~nm} \times 100 \mathrm{~nm} \times 50 \mathrm{~nm}$ ), with a $150 \mathrm{~nm}$ spacing. The SNOM images are for an illumination polarization along $x$ (a) and $y(\mathrm{~b})$. The observed images are reproduced well in the corresponding LDOS images shown in (c) and (d). Figure courtesy of A. Dereux [101].

nanoparticles. Extraordinary images have been produced that reveal a wide variation in the spatial profile of metal nanoparticles as a function of illumination polarization and wavelength. Furthermore, new imaging techniques are now permitting resolution of not just the near-field optical intensity, but the optical phase information as well. Imaging of hot spots on particular types of nanoparticle shapes and the observation of gap modes in nanoparticle assemblies enables further understanding of molecular sensing capabilities and how to optimize this effect. Periodic nanostructures are producing collective plasmon responses and optical near-field responses that can be spatially resolved, realistically facilitating new optical technologies based on photon propagation in nanostructures with sub-wavelength lateral widths. In the future, planarized circuits and waveguides that propagate plasmons rather than electrons or photons are a distinct possibility.

New methods for measuring optical near-fields are sure to develop that will further enable not only the characterization, but the manipulation and control of photons with spatial resolution not possible today. Ultrasmall probes, perhaps single-walled carbon nanotubes, may act as the SNOM probes of tomorrow. Perhaps wholly new methods of imaging will be developed that minimize the need for mechanical probes. For example, photosensitive polymers may function to provide images of optical nearfields by through photo-polymerization or producing mass transport in the illuminated region of the near field of a metal [105]. Ultrahigh resolution confocal microscopy also continues to push to regimes previously not thought possible, particularly with computational analysis and deconvolution of the images [106].
Beyond the particular achievements of the reports summarized here, it is clear that the advent of near-field microscopies is opening a new field, and appreciation for, the interaction of noble metal nanoparticles with light at the nanoscale. The near-field optical properties of noble metal nanoparticles are very rich and diverse, inspiring many scientists in much the same way as the optical properties of noble metal nanoparticles inspired Michael Faraday nearly 150 years ago.

The author thanks Renaud Bachelot, Alexandre Bouhelier, and Gregory Wurtz for stimulating discussions during this writing and assistance with figure preparation. This work was partially supported by the US Department of Energy under contract number W-31-109-ENG-38.

\section{References}

1. L.M. Liz-Marzan, Mater. Today February, 26 (2004)

2. S. Padovani, C. Sada, P. Mazzoldi, B. Brunetti, I. Borgia, A. Sgamellotti, A. Giulivi, F. D'Acapito, G. Battaglin, J. Appl. Phys. 93, 10058 (2003)

3. J. Perez-Arantegui, J. Molera, A. Larrea, T. Pradell, M. Vendrell-Saz, J. Am. Ceram. Soc. 84, 442 (2001)

4. M. Kerker, Appl. Opt. 30, 4699 (1991)

5. M. Faraday, Philos. Trans. Roy. Soc. London 147, 145 (1857)

6. M. Fleischmann, P.J. Hendram, A.J. McQuilla, Chem. Phys. Lett. 62, 163 (1974)

7. D.L. Jeanmaire, R.P. VanDuyne, J. Electroanal. Chem. 84, 1 (1977) 
8. J.A. Creighton, C.G. Blatchford, M.G. Albrecht, J. Chem. Soc. Faraday Trans. 75, 790 (1979)

9. M. Moskovits, Rev. Mod. Phys. 57, 783 (1985)

10. W.E. Doering, S. Nie, J. Phys. Chem. B 106, 311 (2001)

11. N. Felidj, J. Aubard, G. Levi, J.R. Krenn, A. Hohenau, G. Schider, A. Leitner, F.R. Aussenegg, Appl. Phys. Lett. 82, 3095 (2003)

12. C.L. Haynes, R.P.V. Duyne, J. Phys. Chem. B 107, 7426 (2003)

13. U. Kreibig, M. Vollmer, Optical properties of metal clusters (Springer, Berlin, 1995)

14. P.B. Johnson, R.W. Christy, Phys. Rev. B 6, 4370 (1972)

15. G. Mie, Ann. Phys. 26, 329 (1908)

16. J.R. Krenn, A. Dereux, J.C. Weeber, E. Bourillot, Y. Lacroute, J.P. Goudonnet, G. Schider, W. Gotschy, A. Leitner, F.R. Aussenegg, C. Girard, Phys. Rev. Lett. 82, 2590 (1999)

17. S.A. Maier, P.G. Kik, H.A. Atwater, S. Meltzer, E. Harel, B.E. Koel, A.A.G. Requicha, Nat. Mater. 2, 229 (2003)

18. S.A. Maier, M.L. Brongersma, P.G. Kik, S. Meltzer, A.A.G. Requicha, H.A. Atwater, Adv. Mater. 13, 1501 (2001)

19. M. Ohtsu, K. Kobayashi, T. Kawazoe, S. Sangu, T. Yatsui, IEEE J. Quantum Electron. 8, 839 (2002)

20. A.M. Michaels, J. Jiang, L. Brus, J. Phys. Chem. B 104, 11965 (2000)

21. C. Sönnichsen, S. Geier, N.E. Hecker, G. Von Plessen, J. Feldmann, H. Ditlbacher, B. Lamprecht, J.R. Krenn, F.R. Aussenegg, V.Z.-H. Chan, J.P. Spatz, M. Möller, Appl. Phys. Lett. 77, 2949 (2000)

22. R. Jin, Y. Cao, C.A. Mirkin, K.L. Kelly, G.C. Schatz, J.G. Zheng, Science 294, 1901 (2001)

23. J.J. Mock, M. Barbic, D.R. Smith, D.A. Schultz, S. Schultz, J. Chem. Phys. 116, 2755 (2002)

24. T. Klar, M. Perner, S. Grosse, G. v. Plessen, W. Spirkl, J. Feldmann, Phys. Rev. Lett. 80, 4249 (1998)

25. H.F. Hamann, A. Gallagher, D.J. Nesbitt, Appl. Phys. Lett. 73, 1469 (1998)

26. J.P. Kottmann, O.J.F. Martin, Appl. Phys. B 73, 299 (2001)

27. R. Dunn, Chem. Rev. 2891 (1999)

28. D.W. Pohl, Philos. Trans.: Math., Phys., \& Eng. Sci. 362, $701(2004)$

29. D. Courjon, Near-field microscopy and near-field optics (Imperial College Press, London, 2003)

30. Near-field optics and surface plasmon polaritons, edited by S. Kawata (Springer Verlag, 2001), Vol. 81

31. E.H. Synge, Philos. Mag. 6, 356 (1928)

32. E.H. Synge, Philos. Mag. 11, 65 (1931)

33. E.H. Synge, Philos. Mag. 13, 297 (1932)

34. D.W. Pohl, W. Denk, M. Lanz, Appl. Phys. Lett. 44, 651 (1984)

35. H. Raether, Surface plasmons on smooth and rough surfaces and on gratings (Springer-Verlag, Berlin, 1988)

36. J. Wessel, J. Opt. Soc. Am. B 2, 1538 (1985)

37. R. Bachelot, P. Gleyzes, A.C. Boccara, Microsc. Microanal. Microstruct. 5, 389 (1994)

38. Y. Inouye, S. Kawata, J. Microsc. 178, 14 (1994)

39. F. Zenhausern, M.P. O'Boyle, H.K. Wickramasinghe, Appl. Phys. Lett. 66, 1623 (1994)

40. E. Betzig, P.L. Finn, J.S. Weiner, Appl. Phys. Lett. 60, 2484 (1992)
41. R. Bachelot, P. Gleyzes, A.C. Boccara, Probe Microsc. 1, 89 (1997)

42. K. Karrai, D.R. Grober, Appl. Phys. Lett. 66, 1842 (1995)

43. G. Wurtz, R. Bachelot, P. Royer, Eur. Phys. J. Appl. Phys. 5, 269 (1999)

44. E.J. Sanchez, L. Novotny, X.S. Xie, Phys. Rev. Lett. 82, 4014 (1999)

45. A. Bouhelier, M. Beversluis, A. Hartschuh, L. Novotny, Phys. Rev. Lett. 90, 013903/1 (2003)

46. M.R. Beversluis, A. Bouhelier, L. Novotny, Phys. Rev. B 68, 115433/1 (2003)

47. R.C. Reddick, R.J. Warmack, T.L. Ferrell, Phys. Rev. B 39, 767 (1989)

48. D. Courjon, K. Sarayeddine, M. Spajer, Opt. Comm. 71, $23(1989)$

49. F.D. Fornel, J.P. Goudonnet, L. Salomon, E. Lesniewska, in Optical Storage and Scanning Technology, edited by T. Wilson (Proc. SPIE, Bellingham, Washington, 1989), pp. $77-84$

50. B. Hecht, H. Bielefeldt, Y. Inouye, D.W. Pohl, L. Novotny, J. Appl. Phys. 81, 2492 (1997)

51. V.V. Vukovic, J.M. Nedeljkovic, Langmuir 9, 980 (1993)

52. F.X. Redl, K.-S. Cho, C.B. Murray, S. O'Brien, Nature 424, 968 (2003)

53. S.-K. Eah, H.M. Jaeger, N.F. Scherer, X.-M. Lin, G.P. Wiederrecht, Chem. Phys. Lett. 386, 390 (2004)

54. C.L. Haynes, A.D.M.L. Zhao, R.P.V. Duyne, G.C. Schatz, L. Gunnarson, J. Prikulis, B. Kasemo, M. Kall, J. Phys. Chem. B 107, 7337 (2003)

55. Handbook of microlithography, micromachining, and microfabrication, edited by P. Rai-Choudhury (SPIE Optical Engineering Press, London, 1997)

56. U.C. Fischer, D.W. Pohl, Phys. Rev. Lett. 62, 458 (1989)

57. D.P. Tsai, J. Kovacs, Z. Wang, M. Moskovits, V.M. Shalaev, J.S. Suh, R. Botet, Phys. Rev. Lett. 72, 4149 (1994)

58. S. Gresillon, L. Aigouy, A.-C. Boccara, J.-C. Rivoal, X. Quelin, C. Desmarest, P. Gadenne, V.A. Shubin, A.K. Sarychev, V.N. Shalaev, Phys. Rev. Lett. 82, 4520 (1999)

59. S.I. Bozhevolnyi, V.S. Volkov, K. Leosson, Phys. Rev. Lett. 89, 6801/1 (2002)

60. J.R. Krenn, W. Gotschy, D. Somitsch, A. Leitner, F.R. Aussenegg, Appl. Phys. A: Mat. Sci. Eng. 61, 541 (1995)

61. S.A. Maier, P.G. Kik, H.A. Atwater, Appl. Phys. Lett. 81, $1714(2002)$

62. G.A. Wurtz, J.S. Im, S.K. Gray, G.P. Wiederrecht, J. Phys. Chem. B 107, 14191 (2003)

63. B.J. Messinger, K.U. v. Raben, R.K. Chang, P.W. Barber, Phys. Rev. B 24, 649 (1981)

64. W. Rechberger, A. Hohenau, A. Leitner, J.R. Krenn, B. Lamprecht, F.R. Aussenegg, Opt. Comm. 220, 137 (2003)

65. P.M. Adam, S. Benrezzak, J.L. Bijeon, R. Royer, J. Appl. Phys. 88, 6919 (2000)

66. G.A. Wurtz, J. Hranisavljevic, G.P. Wiederrecht, Nano Lett. 3, 1511 (2003)

67. R. Hillenbrand, F. Keilmann, Phys. Rev. Lett. 85, 3029 (2000)

68. A. Nesci, R. Dandliker, H.P. Herzig, Opt. Lett. 26, 208 (2001)

69. S. Aubert, A. Bruyant, S. Blaize, R. Bachelot, G. Lerondel, S. Hudlet, P. Royer, J. Opt. Soc. Am. B 20, 2117 (2003) 
70. R. Hillenbrand, F. Keilmann, Appl. Phys. B 73, 239 (2001)

71. R. Hillenbrand, F. Keilmann, P. Hanarp, D.S. Sutherland, J. Aizpurua, Appl. Phys. Lett. 83, 368 (2003)

72. J. Prikulis, H. Xu, L. Gunnarsson, M. Kall, J. Appl. Phys. 92, $6211(2002)$

73. A.A. Mikhailovsky, M.A. Petruska, K. Li, M.I. Stockman, V.I. Klimov, Phys. Rev. B 69, 08541/1 (2004)

74. A. Mooradian, Phys. Rev. Lett. 22, 185 (1969)

75. A. Bouhelier, M.R. Beversluis, L. Novotny, Appl. Phys. Lett. 83, 5041 (2003)

76. M. Meier, A. Wokaun, P.F. Liao, J. Opt. Soc. Am. B 2, $931(1984)$

77. B. Lamprecht, G. Schider, R.T. Lechner, H. Ditlbacher, J.R. Krenn, A. Leitner, F.R. Aussenegg, Phys. Rev. Lett. 84, $4721(2000)$

78. J.J. Storhoff, A.A. Lazarides, R.C. Mucic, C.A. Mirkin, R.L. Letsinger, G.C. Schatz, J. Am. Chem. Soc. 122, 4640 (2000)

79. J. Kim, K.I.-B. Song, S.Q. Lee, E.U.N.-K. Kim, S.-E.U.L. Choi, Y. Lee, K.-H.O. Park, J. Microsc. 209, 236 (2002)

80. H. Tamaru, H. Kuwata, H.T. Miyazaki, K. Miyano, Appl. Phys. Lett. 80, 1826 (2002)

81. K.-H. Su, Q.-H. Wei, X. Zhang, J.J. Mock, D.R. Smith, S. Schultz, Nano Lett. 3, 1087 (2003)

82. L. Salomon, C. Charbonnier, F.d. Fornel, P.M. Adam, P. Guerin, F. Carcenac, Phys. Rev. B 62, 17072 (2000)

83. M. Quinten, A. Leitner, J.R. Krenn, F.R. Aussenegg, Opt. Lett. 23, 1331 (1998)

84. J. Takahara, S. Yamagishi, H. Taki, A. Morimoto, T. Kobayashi, Opt. Lett. 23, 1331 (1998)

85. R. Bachelot, F. H'Dhili, D. Barchiesi, G. Lerondel, R. Fikri, P. Royer, N. Landraud, J. Peretti, F. Chaput, G. Lempel, J.-P. Boilot, K. Lahlil, J. Appl. Phys. 94, 2060 (2003)

86. L. Novotny, R.X. Bian, X.S. Xie, Phys. Rev. Lett. 79, 645 (1997)

87. Y. Kawata, C. Xu, W. Denk, J. Appl. Phys. 85, 1294 (1999)
88. A. Bouhelier, M. Beversluis, A. Hartschuh, L. Novotny, Phys. Rev. Lett. 90, 13903/1 (2003)

89. N. Richard, J. Appl. Phys. 88, 2318 (2000)

90. N. Richard, Phys. Rev. E 63, 026602/1 (2001)

91. J.P. Kottmann, O.J.F. Martin, D.R. Smith, S. Schultz, Chem. Phys. Lett. 341, 1 (2001)

92. C. Hafner, The Generalized Multiple Multipole Technique for Computational Electromagnetics (Artech, Boston, 1990)

93. L. Novotny, D.W. Pohl, B. Hecht, Opt. Lett. 20, 970 (1995)

94. L. Landau, E. Lifchitz, L. Pitaevskii, Electrodynamics of Continuous Media (Pergamon, Oxford, 1984)

95. J.A. Porto, R. Carminati, J.-J. Greffet, J. Appl. Phys. 88, 4845 (2000)

96. A. Pack, W. Grill, R. Wannemacher, Ultramicroscopy 94, 109 (2003)

97. D.J. Bergman, M.I. Stockman, Phys. Rev. Lett. 90, $027402(2003)$

98. K.R. Li, M.I. Stockman, D.J. Bergman, Phys. Rev. Lett. 91, $227402(2003)$

99. A. Taflove, S.C. Hagness, Computational Electrodynamics: The Finite-Difference Time-Domain Method, 2nd edn. (Artech House, Boston, 2000)

100. S.K. Gray, T. Kupka, Phys. Rev. B 68, 045415 (2003)

101. C. Chicanne, T. David, R. Quidant, J.C. Weeber, Y. Lacroute, E. Bourillot, A. Dereux, G.C. d. Francs, C. Girard, Phys. Rev. Lett. 88, 097402/1 (2002)

102. A. Dereux, C. Girard, C. Chicanne, G.C. d. Francs, T. David, E. Bourillot, Y. Lacroute, J.C. Weeber, Nanotechnology 14, 935 (2003)

103. G.C.d. Francs, C. Girard, J.C. Weeber, C. Chicanne, A. Dereux, D. Peyrade, Phys. Rev. Lett. 86, 4950 (2001)

104. S. John, J. Wang, Phys. Rev. B 43, 12772 (1991)

105. F. H'dhili, R. Bachelot, G. Lerondel, D. Barchiesi, P. Royer, Appl. Phys. Lett. 79, 4019 (2001)

106. B.M. Hanser, M.G.L. Gustafsson, D.A. Agard, J.W. Sedat, Opt. Lett. 28, 801 (2003) 